EDITORIAL

\title{
Challenges in Medical Education: A Long Way up to Meet
}

\author{
*M. Rahman \\ Prof. M. Rahman, Head, Department of Community Medicine, AKMMC \\ *Corresponding Author
}

Bangladesh is continuing with disease burden like; malnutrition, communicable \& non-communicable diseases along with mental and psycho-social problems including many emerging \& re-emerging diseases. This is however, aggravated by natural \& manmade disasters and conflicts. Moreover, there is a problem of access of large sector of population to food security, safe water (arsenic contamination), clean environment, adequate wage and healthy work environment. There are social obligations of efficient and accessible health care delivery services too. Recognizing all those challenges and social demands it is a felt need of new paradigms \& new understanding of preparing future graduates as a comprehensive health care providers with multifaceted roles \& responsibilities.

The challenges in medical education in Bangladesh includes rapid growth of medical colleges, questionable student selection policies, weakly focused curriculum on health care needs, one national undergraduate medical curriculum with significant deficiency in internship training implementation, and lack of need based faculty development program and also the utilization of graduates to serve the large segment of rural population. Despite of many changes advocated by BMDC and CME for the medical institutions in the country those are moving slowly and not getting shape accordingly perhaps due to commercialization of education. Therefore, it is needed to implement quality assurance in medical education \& health care delivery along with accreditation mechanism which is still thriving to achieve with minimum effort.

Health is a state of complete physical, mental and social well being in addition to moral as well. It is influenced by many factors related to politics, economics, education, culture and environment in particular. Thus it is essential to create a link of cooperation with all those relevant factors. On the other hand, society wants to respond people's health needs with such interventions that are relevant, efficient, affordable and equitable to them. Therefore, each and every nation should respond to reform their health systems and education in a way to achieve those goals relevant \& effective in meeting social needs in order to address newer challenges towards socially and economically productive life of the population at large.

The country demands undergraduate curriculum reforms relevant to social needs $\&$ be practiced by individual University. The content of the curriculum and teaching learning settings must be designed appropriately to develop knowledge, skills \& attitudes to meet challenges \& social needs towards better health. Thus curriculum redesign should emphasize social and clinical needs in addition to teaching learning settings with problem-solving and evidence based approach. Each institution should have a compulsory established outreach centre for its own and be utilized by the students \& interns as a teaching learning setting under guidance of relevant disciplines. It will open an opportunity to work in a real environment for providing services and to meet the needs of the rural people as well. Moreover, formative assessment should carry more emphasis over summative. The assessment by Multiple Choice Question (MCQ), Objective Structured Practical Examination (OSPE) and Objective Structured Clinical Examination (OSCE) needs to be designed emphasizing more on criterion-reference standards. Nevertheless, improved vertical integration through problem-based organ system design curriculum may help addressing these issues as well. In addition internship training should be more on skill oriented with meaningful supervision and assessment. The final crucial issue is the faculty development although in progress by medical education unit in different institutions however, it must put as a predominant institutional affiliation criteria for its proper functional contribution. The implementation of institutional quality assurance \& accreditation mechanism can also be considered in the same direction. 